\title{
The sentinel node procedure in colon carcinoma: a multi-centre study in The Netherlands
}

\author{
Wendy Kelder • Andries E. Braat • Arend Karrenbeld • \\ Joris A. K. Grond • Johannes E. De Vries • \\ J. Wolter A. Oosterhuis • Peter C. Baas • \\ John T. M. Plukker
}

Accepted: 15 June 2007 / Published online: 12 July 2007

(C) Springer-Verlag 2007

\begin{abstract}
Background Lymph node status is the most important predictive factor in colorectal carcinoma. Recurrences occur in $20 \%$ of the patients without lymph node metastases. The sentinel lymph node (SLN) biopsy is a tool to facilitate identification of micrometastatic disease and aberrant lymphatic drainage. We studied the feasibility of in vivo SLN detection in a multi-centre setting and evaluated nodal micro-staging using immunohistochemistry (IHC).

Materials and methods Sub-serosal injection with Patent Blue dye was used in the SLN procedure in 69 patients
\end{abstract}

Both authors Wendy Kelder and Andries E. Braat contributed equally to this study.

W. Kelder· P. C. Baas

Department of Surgery, Martini Hospital,

Groningen, The Netherlands

W. Kelder · J. T. M. Plukker $(\bowtie)$

Department of Surgery, University Medical Centre Groningen,

Hanzeplein 1, 9700 RB,

Groningen, The Netherlands

e-mail: j.t.m.plukker@chir.umcg.nl

A. E. Braat • J. E. De Vries · J. W. A. Oosterhuis

Department of Surgery, Isala Klinieken,

Zwolle, The Netherlands

A. Karrenbeld

Department of Pathology, University Medical Centre,

Groningen, The Netherlands

J. A. K. Grond

Department of Pathology, Laboratory of Public Health,

Leeuwarden, The Netherlands

A. E. Braat

Department of Surgery, Leiden University Medical Centre,

Leiden, The Netherlands operated for localized colon cancer in six Dutch hospitals. Each SLN was examined with routine haematoxylin-eosin staining. In tumour-negative SLNs, we performed CK $7 / 8$ or 18 IHC.

Results The procedure was successful in 67 of 69 patients (97\%). The SLN was negative in 43 patients. In three cases, it was false negative, resulting in a negative predictive value of $93 \%$ and an accuracy of $96 \%$. In 24 of 27 patients with lymph node metastases in a successful SLN procedure, the SLN was positive (sensitivity 89\%). In 15 patients, the SLN was the only positive node (21\%). In nine patients, we only found micrometastases or isolated tumour cells, resulting in $18 \%$ upstaging. Aberrant lymphatic drainage was seen in three patients (4\%).

Conclusion The SLN procedure in localized colon carcinoma is reliable in a multi-centre setting. It is helpful to identify patients who would be classified as stage II with conventional staging (18\%) and who might benefit from adjuvant treatment.

Keywords Colon carcinoma - Sentinel lymph node . Micrometastasis $\cdot$ Minimal residual disease

\section{Introduction}

Survival in patients with colon carcinoma is strongly correlated with lymph node status: the 5-year disease-free survival rate is $70-80 \%$ for patients with lymph nodenegative disease (stage I/II) but only $45-50 \%$ for those with node-positive disease (stage III) [1]. The presence of lymph node metastases indicates the use of adjuvant chemotherapy in these patients, which increases the 5-year survival rate with about $10 \%$ [2]. Despite the favourable prognosis of patients with localized colon carcinoma without regional 
lymph node metastasis, $20-30 \%$ of these patients will develop recurrent disease after apparently curative resection [3]. It is possible that in this group of patients, small lymph node metastases have been missed, resulting in understaging. This may be due to an inadequate surgical lymphadenectomy or insufficient pathological examination [4]. According to international guidelines, meticulous pathological examination of at least 12 lymph nodes is warranted for adequate staging of patients with colon carcinoma [5]. However, several studies showed that the minimal number of lymph nodes necessary for correct staging varied considerably from 6 to 18 to as many as possible in the study of Goldstein et al. [4, 6-9]. In addition, in-depth pathological examination of lymph nodes by immunohistochemical staining for cytokeratin or reverse transcriptase polymerase chain reaction (RT-PCR) may reveal micrometastases that could have been missed by routine haematoxylin and eosin (H\&E) examination. Several authors have reported a decreased survival rate when micrometastases are detected in colon carcinoma [10-13]. The possible benefit of adjuvant therapy in this group of patients is not clear yet.

These (ultra-)staging techniques are time consuming, labour intensive and costly. For optimal staging, in depth examination of only the sentinel lymph node (SLN) could be helpful. In colon carcinoma, the SLNs are defined as the first one to four blue-stained nodes with the most direct lymph drainage from the primary tumour. They have the greatest potential to harbour metastatic disease when present, enabling focussed examination with multi-level micro-sectioning of the SLNs to provide a more efficient and cost-effective detection of micrometastases. In addition, patterns of aberrant lymphatic drainage can be visualized with SLN mapping, which may lead to a more extended resection. Several studies have reported varying results of the SLN procedure in colon carcinoma [14-20]. This study presents the results of the SLN procedure in six Dutch hospitals. The primary aim of this study is to test the accuracy and sensitivity of the SLN procedure in a multicentre setting. Furthermore, we looked at upstaging and possible aberrant lymphatic drainage.

\section{Materials and methods}

This study was performed between May 2002 and May 2005 in five teaching hospitals and one university hospital. All procedures were supervised by one of the coordinating surgeons (Plukker, Braat). Only patients with histologically proven primary colon carcinoma were included in the study. Patients with distant metastases or gross lymph node involvement as shown by pre-operative examinations or palpation during surgery were excluded. The procedure was only performed when one of the study coordinators was available for supervision (Plukker, Braat, Kelder). The study was approved by the local scientific ethics committee, and all patients had given informed consent. Patients with rectal cancer were excluded from the study.

SLN mapping was carried out through an open procedure by injection of 1-3 ml Patent Blue with a tuberculin syringe and 29-gauge needle sub-serosally in four quadrants around the tumour. The sub-serosal injection was carried out before vascular ligation. Within 5 to $10 \mathrm{~min}$ after the blue dye injection, the SLN could be identified by following the blue-stained lymphatic vessels leading to the blue-stained SLN. These lymph nodes were tagged with a long suture. SLNs were defined as the first one to four bluestained lymph nodes seen within the regional basin. After marking of the SLNs, routine resection was performed. If the SLN was found outside the normal lymphatic basin, we performed an extended resection. The tumour and all lymph nodes were examined according to standard guidelines [5]. If the SLNs were negative after routine H\&E staining, they were sectioned at $150-\mu \mathrm{m}$ intervals and examined at three levels with $\mathrm{H} \& \mathrm{E}$ as well as immunohistochemistry on cytokeratins (CK7/8 or 18). Metastases between 0.2 and $2 \mathrm{~mm}$ were referred to as micrometastases. Metastases smaller than $0.2 \mathrm{~mm}$ were referred to as isolated tumour cells [5]. Upstaging was defined as the presence of micrometastases or isolated tumour cells after immunohistochemistry (IHC) in patients with a negative lymph node status after H\&E.

\section{Definitions}

See Fig. 1. Identification rate is the number of patients with one or more SLNs identified (b)/the total number of procedures (a) $\times 100 \%$. Negative SLNs were false negative if one of the other regional lymph nodes (non-SLNs) were tumour positive (d). The accuracy of the SLN procedure suggests a conformity of the SLN status and the regional nodal status, i.e. the total number of patients with a positive SLN (c)+the number of patients with a true-negative SLN (e)/the number of patients with an identified SLN (b) $\times 100 \%$. Sensitivity is the number of patients with a positive SLN (c)/ The total number of node positive patients $(c+d) \times 100 \%$. Upstaging is the number of patients with positive SLNs by IHC (g)/the number of patients who were node negative by $\mathrm{H} \& \mathrm{E}$ examination $(\mathrm{e}+\mathrm{g}) \times 100 \%$.

\section{Results}

The SLN procedure was performed in 69 patients. Tumour characteristics are shown in Table 1. Figure 1 shows the 


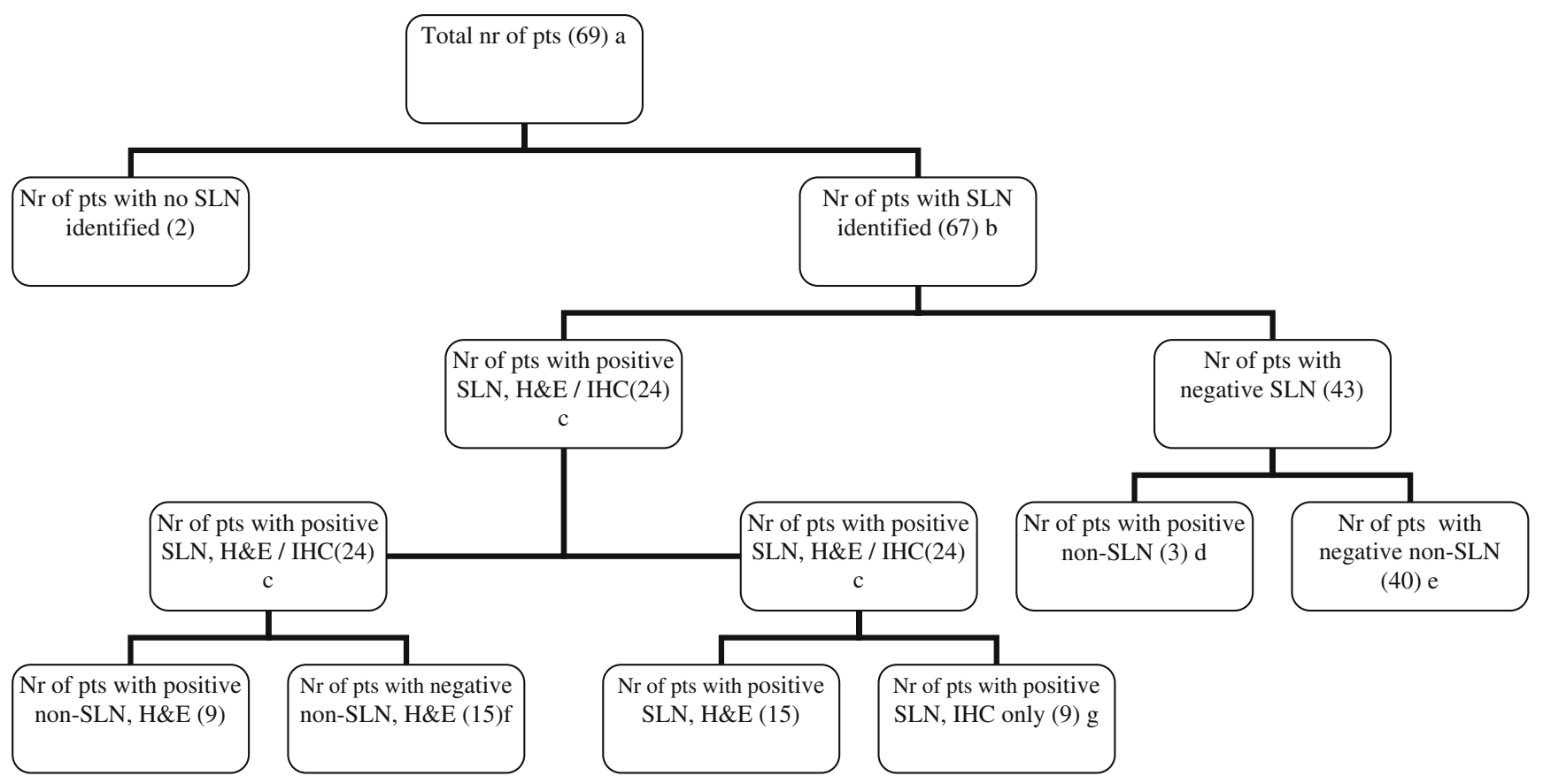

Fig. 1 Flowchart. SLN Sentinel lymph node, pts patients

total number of patients and SLN results. At pathological examination, a mean of 11 nodes per specimen was found; per hospital, this varied between 9 and $17(9,10,12,14,17$, 17, respectively). The mean number of SLNs was 2.3 per patient. The SLN was identified in 67 out of 69 patients (97\%). One of the two failed procedures was in a patient with a carcinoma in the sigmoid colon surrounded by a concurrent diverticulitis. The other patient had extended lymph node metastases with angio-invasion at pathological examination. In 28 patients, lymph node metastases were identified at the pathological examination; this includes the one case where the SLN procedure failed because of extensive lymph node metastases (28 of $69,41 \%$ ). This one case with lymph node metastases and a failed procedure was excluded from further statistical analysis on the SLN procedure, leaving 27 node-positive patients in

Table 1 Tumour characteristics

\begin{tabular}{ll}
\hline Characteristics & Values \\
\hline Tumour location & \\
Right colon & 35 \\
Left colon & 2 \\
Sigmoid colon & 32 \\
T-stage & \\
1 & 1 \\
2 & 14 \\
3 & 48 \\
4 & 6 \\
Mean number of lymph nodes & 11 \\
Mean number of SLN & 2.3 \\
\hline
\end{tabular}

the final analysis. In 24 patients, the SLN was positive, either with H\&E staining or with IHC, resulting in a sensitivity of $89 \%$ ( 24 of 27 ) in the group of 67 patients with a successful SLN procedure. If we leave out the patients who had a positive sentinel node only after IHC, the sensitivity is 15 of 18 (83\%). In 15 of 24 SLN-positive patients, the SLN was the only involved lymph node (63\%). In 9 of 27 lymph node-positive patients, metastases were found only after IHC. In four patients, these were micrometastases, whereas in five cases, isolated tumour cells were found. Therefore, without IHC, the number of nodepositive cases would have been 27 minus 9 , which is 18 . This corresponds to a total of 49 node-negative cases by $\mathrm{H} \& \mathrm{E}$ in the group with a successful SLN procedure. With IHC, the upstaging is 9 of 49 or $18 \%$. The SLN was negative in 43 patients. In 40 patients, the non-SLNs were also negative. This results in a negative predictive value of $93 \%$ (40 of 43). One of the three patients with a falsenegative SLN had lymph node metastases with extra-nodal growth in the non-SLN. In another patient, a small tumour deposit was found in the mesocolon right next to the primary tumour. This was classified as N1 according to the American Joint Committee on Cancer classification, although it is unclear whether this is a true lymph node metastasis or some kind of 'in transit' metastasis. The last patient with a positive non-SLN showed micrometastases at H\&E examination in a small peritumoural lymph node. Aberrant lymphatic drainage was seen in three patients (4\%). In two cases, the SLN was found on the left side of the middle colic artery in patients with a tumour in the ascending colon. In both cases, an extended right hemi- 
colectomy was performed. The third patient had a tumour near the rectosigmoid junction with a high para-aortal SLN. Therefore, we performed an extended left sided resection en-bloc with a partial para-aortal dissection. None of these lymph nodes contained metastases. All other SLNs were found in the mesocolon in close proximity to the tumour. In these cases, the central lymph node as identified by the pathologist was always a non-SLN. The accuracy of the SLN procedure in this study was $96 \%$, as the pathological status of the SLN corresponds with the definitive lymph node status in 64 of the 67 patients.

\section{Discussion}

With an identification rate of $97 \%$, accuracy of $96 \%$, sensitivity of $89 \%$ and negative predictive value of $93 \%$, this study shows that it is possible to perform the SLN procedure properly in patients with localized colon carcinoma in a multi-centre setting. Other multi-centre studies showed varying results of this technique (Table 2) [14-21]. Our results correlate with those from other larger studies, which show accuracy and sensitivity rates of 95-98 and 89-93\%, respectively [15, 18-20]. Most smaller studies show worse results with low accuracy and success rates and corresponding low sensitivity rates and negative predictive values $[14,17,21]$. In one study, the time between injection of the blue dye and identifying the SLN was too long, leading to a larger number of SLNs [17]. It is very likely that not all of these blue nodes were true SLNs. In the study by Bertagnolli et al. [14], 79 patients were operated on by 25 different surgeons in 13 different hospitals. A mean of three procedures per surgeon seems insufficient to adequately learn this technique. It is known that the learning curve of the SLN in colon carcinoma stabilizes after about five procedures [20]. To minimize technical failures, the procedure in our study was performed by a few surgeons under direct supervision of one of the two surgeons coordinating this study (Braat, Plukker). Apart from too few procedures, the worse results in some studies might be explained by inclusion of patients with advanced disease. Some studies included patients with clinically apparent stage III or stage IV disease [21]. Widespread lymph node metastases could result in obstruction of lymphatic channels, and lymphatic drainage is bypassed to other (non-sentinel) lymph nodes. This phenomenon is called skip-metastasis. It was noted in one of the patients with a false-negative SLN in our study who had advanced lymphatic metastases with extra-nodal growth. Patient selection is therefore important for a reliable SLN procedure in colon carcinoma. In fact, the SLN procedure is not useful in patients with clinically apparent stage III or stage IV disease as false-negativity rates will be higher. Moreover, in these patients, metastases will be easily found at routine pathological examination, and the SLN procedure will not have any additional value. The SLN procedure could be useful in those patients with (micro) metastases that would not be identified with routine pathological examination. Furthermore, the failed procedure in one of our patients with concurrent diverticulitis also suggests the importance of an undisturbed lymphatic drainage for a successful SLN procedure.

We saw aberrant lymphatic drainage in three patients (4\%). This percentage correlates with the literature $[15,18$, 20]. In this study, none of these aberrant SLNs showed metastases. However, potentially, these aberrant SLNs are the only lymph nodes containing metastasis, as shown in a previous study [22]. In an experimental situation, it seems justified to perform an extended resection in these cases. Further study should be performed to justify an extended resection in the daily practice.

Literature not clearly indicates how many nodes should be examined to accurately predict lymph node status [6-8, $23,24]$. One study showed that a colon specimen usually contains about 50 lymph nodes and that more than $70 \%$ of the lymph nodes containing metastases are smaller than $5 \mathrm{~mm}$ [9]. It is also known that the prognosis in nodenegative patients with colon carcinoma is better when more lymph nodes have been examined [24]. Taking this into account, the pathologist takes only a sample of the lymphatic basin of a resected colon specimen, even when international guidelines are followed, which state that at least 12 lymph nodes are needed for adequate staging [5]. The mean number of 11 lymph nodes in our study is not enough to predict lymph node status according to the international guideline. This fact could theoretically lower

Table 2 Results of multi-centre studies of the SLN procedure in colon cancer

\begin{tabular}{|c|c|c|c|c|c|c|}
\hline Study & Number of patients & Number of centres & Identification rate $(\%)$ & $\begin{array}{l}\text { Accuracy } \\
(\%)\end{array}$ & $\begin{array}{l}\text { Sensitivity } \\
(\%)\end{array}$ & $\begin{array}{l}\text { Upstaging } \\
(\%)\end{array}$ \\
\hline Bilchik et al. [15] & 40 & 3 & 100 & 100 & 100 & 10 \\
\hline Saha et al. [18] & 131 & 3 & 99 & 97 & 92 & 16 \\
\hline Bertagnolli et al. [14] & 72 & 13 & 92 & 81 & 42 & 0 \\
\hline Read et al. [21] & 38 & 2 & 79 & 76 & 25 & 3 \\
\hline Kelder and Braat & 69 & 6 & 97 & 96 & 89 & 13 or 18 \\
\hline
\end{tabular}


the chance to detect metastases in non-SLN and thus could lower the false-negative rate. However, we did not find any differences in false negative rates between the two hospitals with a mean number of nine and ten examined nodes (40 cases) and the hospitals with more than 12 examined nodes (29 cases). With regards to upstaging, most studies show an upstaging of $10-16 \%[15,18-20]$. However, they calculated upstaging by dividing the number of IHC-positive patients by the total number of patients (Fig. 1g/a, 9 of $69,13 \%$ in our group). We think it is better to consider upstaging solely in the H\&E node-negative group, as this is the group to be upstaged by IHC. Using this method, we find $18 \%$ upstaging in our series. In addition to this true upstaging, patients with a SLN as the only site of metastases could have been 'possibly upstaged,' as conventional pathological dissection of the mesentery might have missed this lymph node. The SLN procedure with patent blue might be able to improve adequacy of the lymph node examination by selecting the right lymph nodes, even small nodes less than $5 \mathrm{~mm}$, to be examined in depth by the pathologist. We found the SLN to be the single lymph node with metastasis in $15(21 \%)$ of the patients (Fig. 1 f). 'Possible upstaging' might play a role here, but we cannot prove this.

As we believe that even isolated tumour cells are important for staging, we assigned patients with micrometastases or isolated tumour cells to the group of nodepositive patients. It must be remarked, however, that these cases were also used for the calculation of upstaging. Our idea of the biological importance of micrometastases and isolated tumour cells is based on a recent meta-analysis that showed that micrometastases detected retrospectively by RT-PCR correlated better with overall survival than IHC and carried significant prognostic value [12].

Regarding the detection of micrometastases, two studies showed a high reliability of the SLN concept to predict micrometastases and/or isolated tumour cells also in nonSLNs. Therefore, it seems sufficient to perform IHC only on the SLN, while examining the non-SLNs with H\&E $[25,26]$. Prospective studies are needed to evaluate the potential benefit of systemic chemotherapy in patients with these micrometastases. A reliable SLN procedure might facilitate this intensive pathological examination by allowing focussed examination of only the SLN and thereby aid in a better patient selection for adjuvant therapy in the future.

\section{References}

1. Hermanek P (1995) pTNM and residual tumor classifications: problems of assessment and prognostic significance. World J Surg 19:184-190
2. Hermanek P (1995) Efficacy of adjuvant fluorouracil and folinic acid in colon cancer. International Multicentre Pooled Analysis of Colon Cancer Trials (IMPACT) investigators. Lancet 345:939944

3. Wolmark N, Fisher B, Wieand HS (1986) The prognostic value of the modifications of the Dukes' C class of colorectal cancer. An analysis of the NSABP clinical trials. Ann Surg 203:115-122

4. Joseph NE, Sigurdson ER, Hanlon AL, Wang H, Mayer RJ, MacDonald JS, Catalano PJ, Haller DG (2003) Accuracy of determining nodal negativity in colorectal cancer on the basis of the number of nodes retrieved on resection. Ann Surg Oncol 10:213-218

5. Greene FL, Page DL, Fleming ID et al (2002) American Joint Committee on Cancer - cancer staging handbook, TNM classification of malignant tumors, 129. Springer, New York, NY

6. Fielding LP, Arsenault PA, Chapuis PH, Dent O, Gathright B, Hardcastle JD, Hermanek P, Jass JR, Newland RC (1991) Clinicopathological staging for colorectal cancer: an International Documentation System (IDS) and an International Comprehensive Anatomical Terminology (ICAT). J Gastroenterol Hepatol 6:325344

7. Goldstein NS (2002) Lymph node recoveries from 2427 pT3 colorectal resection specimens spanning 45 years: recommendations for a minimum number of recovered lymph nodes based on predictive probabilities. Am J Surg Pathol 26:179-189

8. Hernanz F, Revuelta S, Redondo C, Madrazo C, Castillo J, Gomez-Fleitas M (1994) Colorectal adenocarcinoma: quality of the assessment of lymph node metastases. Dis Colon Rectum 37:373-376

9. Haboubi NY, Abdalla SA, Amini S, Clark P, Dougal M, Dube A, Schofield P (1998) The novel combination of fat clearance and immunohistochemistry improves prediction of the outcome of patients with colorectal carcinomas: a preliminary study. Int J Colorectal Dis 13:99-102

10. Greenson JK, Isenhart CE, Rice R, Mojzisik C, Houchens D, Martin EW Jr (1994) Identification of occult micrometastases in pericolic lymph nodes of Duke's B colorectal cancer patients using monoclonal antibodies against cytokeratin and CC49. Correlation with long-term survival. Cancer 73:563-569

11. Liefers GJ, Cleton-Jansen AM, Velde CJ, Hermans J, Krieken JH, Cornelisse CJ, Tollenaar RA (1998) Micrometastases and survival in stage II colorectal cancer. N Engl J Med 339:223-228

12. Iddings D, Ahmad A, Elashoff D, Bilchik A (2006) The prognostic effect of micrometastases in previously staged lymph node negative (N0) colorectal carcinoma: a meta-analysis. Ann Surg Oncol 13:1386-1392

13. Noura S, Yamamoto $H$, Ohnishi $T$, Masuda N, Matsumoto $T$, Takayama O, Fukunaga H, Miyake Y, Ikenaga M, Ikeda M, Sekimoto M, Matsuura N, Monden M (2002) Comparative detection of lymph node micrometastases of stage II colorectal cancer by reverse transcriptase polymerase chain reaction and immunohistochemistry. J Clin Oncol 20:4232-4241

14. Bertagnolli M, Miedema B, Redston M, Dowell J, Niedzwiecki D, Fleshman J, Bem J, Mayer R, Zinner M, Compton C (2004) Sentinel node staging of resectable colon cancer: results of a multicenter study. Ann Surg 240:624-628

15. Bilchik AJ, Saha S, Wiese D, Stonecypher JA, Wood TF, Sostrin S, Turner RR, Wang HJ, Morton DL, Hoon DS (2001) Molecular staging of early colon cancer on the basis of sentinel node analysis: a multicenter phase II trial. J Clin Oncol 19:11281136

16. Braat AE, Oosterhuis JW, Moll FC, Vries JE (2004) Successful sentinel node identification in colon carcinoma using Patent Blue V. Eur J Surg Oncol 30:633-637

17. Joosten JJ, Strobbe LJ, Wauters CA, Pruszczynski M, Wobbes T, Ruers TJ (1999) Intraoperative lymphatic mapping and the 
sentinel node concept in colorectal carcinoma. Br J Surg 86:482486

18. Saha S, Nora D, Wong JH, Weise D (2000) Sentinel lymph node mapping in colorectal cancer-a review. Surg Clin North Am 80:1811-1819

19. Tsioulias GJ, Wood TF, Spirt M, Morton DL, Bilchik AJ (2002) A novel lymphatic mapping technique to improve localization and staging of early colon cancer during laparoscopic colectomy. Am Surg 68:561-565

20. Paramo JC, Summerall J, Poppiti R, Mesko TW (2002) Validation of sentinel node mapping in patients with colon cancer. Ann Surg Oncol 9:550-554

21. Read TE, Fleshman JW, Caushaj PF (2005) Sentinel lymph node mapping for adenocarcinoma of the colon does not improve staging accuracy. Dis Colon Rectum 48:80-85

22. Bilchik AJ, Saha S, Tsioulias GJ, Wood TF, Morton DL (2001) Aberrant drainage and missed micrometastases: the value of lymphatic mapping and focused analysis of sentinel lymph nodes in gastrointestinal neoplasms. Ann Surg Oncol 8:82S-85S

23. Wong JH, Severino R, Honnebier MB, Tom P, Namiki TS (1999) Number of nodes examined and staging accuracy in colorectal carcinoma. J Clin Oncol 17:2896-2900

24. Le Voyer TE, Sigurdson ER, Hanlon AL, Mayer RJ, MacDonald JS, Catalano PJ, Haller DG (2003) Colon cancer survival is associated with increasing number of lymph nodes analyzed: a secondary survey of intergroup trial INT-0089. J Clin Oncol 21:2912-2919

25. Turner RR, Nora DT, Trocha SD, Bilchik AJ (2003) Colorectal carcinoma nodal staging. Frequency and nature of cytokeratinpositive cells in sentinel and nonsentinel lymph nodes. Arch Pathol Lab Med 127:673-679

26. Bembenek A, Schneider U, Gretschel S, Fischer J, Schlag PM (2005) Detection of lymph node micrometastases and isolated tumor cells in sentinel and nonsentinel lymph nodes of colon cancer patients. World J Surg 29:1172-1175 